\title{
Investigation on Biogenic Amines in Plant-based Minor Korean Fermented Foods
}

\author{
Jin Hyo Kim* · Sung-Ji Ryu · Ji-Won Lee · Young-Wan Kim · Han-Joon Hwang \\ - Oh-Kyoung Kwon
}

\section{소규모 국내생산 식물 발효식품의 바이오제닉아민 잔류특성}

김진효* · 류성지 · 이지원 · 김영완 · 황한준 · 권오경

Received: 4 December 2012 / Accepted: 12 March 2013 / Published Online: 30 June 2013

(C) The Korean Society for Applied Biological Chemistry 2013

\begin{abstract}
Ten major residual biogenic amines including toxic histamine and tyramine were investigated in the plant-based minor Korean fermented food. From the analyses of pickled vegetables, fermented vegetable extracts, fermented tea, black garlic and herbal rice wines, more than $100 \mathrm{mg} / \mathrm{kg}$ of histamine were found in pickled soy leaf and pickled mulberry leaf, and also over 1,000 $\mathrm{mg} / \mathrm{kg}$ of total biogenic amines were found in pickled soy leaf. No sample was found over in black garlic, fermented tea, fermented vegetable extracts and herbal rice wine, less than $100 \mathrm{mg} / \mathrm{kg}$ of histamine and/or $1,000 \mathrm{mg} / \mathrm{kg}$ of total biogenic amines were observed. Interestingly, all the tested rice wines were found to be over $100 \mathrm{mg} / \mathrm{kg}$ of agmatine residue.
\end{abstract}

Keywords biogenic amine $\cdot$ histamine $\cdot$ Korean fermented food - quantitative analysis

\section{서 론}

바이오제닉아민(BAs)은 미생물, 식물, 동물의 대사과정 중 아미 노산의 decarboxylation 혹은 알데하이드나 케톤의 transamination 을 통해 생성되며(Karovicova and Kohajdova, 2005), 화학구조

J. H. Kim · S. -J. Ryu · J. -W. Lee · O. -K. Kwon

Chemical Safety Division, National Academy of Agricultural Science, Rural Development Administration, Suwon 441-707, Republic of Korea

Young-Wan Kim · Han-Joon Hwang

Department of Food and Biotechnology, Korea University, Sejong, 339700 , Republic of Korea

*Corresponding author (J. H. Kim: setup75@korea.kr)
를 바탕으로 aliphatic BAs (putrescine, cadaverine, spermine, spermidine), aromatic BAs (tyramine, phenylethylamine), heterocyclic BAs (histamine, tryptamine)으로 구분하기도 한다(Silla-Santos, 1996) (Fig. 1). 이러한 BAs은 호르몬, 알카로이드, 핵산, 단백 질 등의 생합성을 위한 전구물질로 알려져 있으며, 체온 및 혈 압조절 등의 기능을 갖는 것으로 보고되었다(Silla-Santos, 1996; Greif 등, 1997). 또한, putrescine, spermidine, spermine은 세포 분화, 개화, 외부 스트레스 대응과 같은 기능 및 식품의 향과 맛에도 영향을 주는 것으로 알려져 있다(Halasz 등, 1994). 하 지만, 이러한 $\mathrm{BAs}$ 의 긍정적인 역할 외에도 putrescine, spermidine, spermine은 종양생장을 촉진하며(Bardocz, 1995), agmatine, spermine, spermidine 등은 발암물질인 nitrosamine의 전구체로 알려져 있다(Halasz 등, 1994). 특히, histamine은 scombrotoxicosis 를 유발하는 것으로 알려져 있으며, tyramine은 혈관수축에 관 여하여 'cheese reaction'을 통한 고혈압 및 편두통을 유발하는 것으로 알려져 있다(Bardocz, 1995; Cho 등, 2006). 이러한 독 성으로 인해, 미국 FDA는 수산물에서 histamine $50 \mathrm{mg} / \mathrm{kg}$, tyramine $100 \mathrm{mg} / \mathrm{kg}$, 총 BAs $1,000 \mathrm{mg} / \mathrm{kg}$ 을 수산식품의 잔류허 용기준으로, EU에서는 histamine $100 \mathrm{mg} / \mathrm{kg}$, 총 BAs $300 \mathrm{mg}$ / $\mathrm{kg}$ 을 수산물의 잔류 허용 기준으로 제안하고 있음에도 불구하고 (Lehane and Olley, 2000; Hu 등, 2012) 아직 국내에서는 $\mathrm{BAs}$ 관련 기준이 마련되어 있지 않은 실정이다. 하지만, 국내 에서도 histamine과 tyramine을 중심으로 한 BAs 잔류모니터링 및 위해성평가 연구가 수산물 및 발효식품을 중심으로 이루어 지고 있으며(Beneduce 등, 2010; Chong 등, 2011; Hu 등, 2012), 국내의 경우 장류를 포함하는 주요 발효 농수산식품에 대한 BAs의 저감기술 연구도 진행되고 있다(Kim 등, 2002; Choi 등, 2012).

지금까지의 $\mathrm{BAs}$ 잔류모니터링 결과를 종합하면, 주스류에서 putrescine, cadaverine, histamine, tyramine이 잔류하는 것으로 확인되었으며, 주류 및 김치류, 장류 등에서도 다양한 종류의 
<smiles>NCCCCN</smiles><smiles>NCCCNCCCCNCCCN</smiles>
Spermine<smiles>NCCCCCN</smiles>

Cadaverine<smiles>NCCCCNCCCN</smiles><smiles>NCCc1ccc(O)cc1</smiles>

Tyramine<smiles>NCCc1c[nH]c2ccc(O)cc12</smiles>
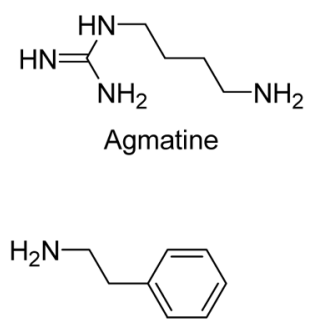

$\beta$-Phenylethylamine

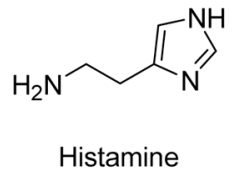

Fig. 1 Tested ten biogenic amines.<smiles>NCCc1c[nH]c2ccccc12</smiles>

Tryptamine

Serotonin

BAs가 잔류하는 것으로 보고된 바 있다(Karovicova and Kohajdova, 2005; Cho 등, 2006). 하지만, 국내의 다양한 발효식품제조 특 성상 식품 내 생성잔류 가능한 BAs 함량이 일정하지 않음에 따라 지속적인 모니터링 검사 및 관리가 필요하지만, 주요 발 효식품류인 장류, 김치류를 제외한 소규모 발효식품군에서의 $\mathrm{BAs}$ 잔류분석 연구가 부족하여, 본 연구에서는 이를 보완하고 자 비주류 발효식품군에서의 BAs 잔류특성을 분석하였다.

\section{재료 및 방법}

시료준비. 본 연구에 사용된 식물 발효식품은 시중에 유통되고 있는 제품으로 옻나무, 매실, 도라지, 오미자, 블루베리, 석류, 솔잎, 복분자, 오디, 산머루를 이용한 진액류 30 종, 솔잎, 울금, 가시오가피, 인삼 등을 첨가하여 제조한 막걸리 14종, 깻잎, 뽕 잎, 콩잎, 고추, 마늘, 감, 양파, 더덕, 참외, 매실로 제조한 장 아찌 28 종, 흑마늘 10 종, 발효차 15 종을 각각 제조사를 달리하 여 시장에서 구매하여 사용하였다.

표준물질 및 시약. 시험에 사용된 $\mathrm{BAs}$ 의 표준물질 agmatine (AGM) sulfate, tryptamine (TRP), $\beta$-phenylethylamine (PHE) hydrochloride, putrescine (PUT) dihydrochloride, cadaverine (CAD), histamine (HIS) dihydrochloride, serotonine (SER), tyramine (TYR), spermidine (SPD), spermine (SPM), 1,7diaminoheptane (IS)과 유도체화 시약인 dansyl chloride를 Sigma-Aldrich Co. (Steinhein, Germany)에서 구매하였다. $\mathrm{Na}_{2} \mathrm{CO}_{3}$, 무수 $\mathrm{Na}_{2} \mathrm{SO}_{4}, 37 \% \mathrm{HCl}$ 과 용매로 사용된 HPLC등급 의 acetone, acetonitrile, diethyl ether는 Merck Co. (KGaA, Germany) 제품을 사용하였다.

시료전처리. 발효차를 제외한 모든 시료는 Cho 등(2006)이 사 용한 방법을 수정하여 다음과 같이 수행하였다. 액상 및 고상 시료 $5 \mathrm{~g}$ 을 $0.1 \mathrm{~N} \mathrm{HCl} 20 \mathrm{~mL}$ 를 넣고 균질화 시킨 후, 원심분 리 $\left(3,000 \mathrm{rpm}, 4^{\circ} \mathrm{C}, 20 \mathrm{~min}\right)$ 하여 상등액을 취한다. 침전물에 $0.1 \mathrm{~N} \mathrm{HCl}(20 \mathrm{~mL} \times 2)$ 을 다시 가한 후 위 조작을 반복하여 얻 은 총 추출용액의 부피가 $50 \mathrm{~mL}$ 가 되도록 하였다. 발효차는 0.1 $\mathrm{N} \mathrm{HCl} 100 \mathrm{~mL}$ 에 시료 $5 \mathrm{~g}$ 을 넣고 $80^{\circ} \mathrm{C}$ 에서 4 시간 동안 추출 하였다. 시료 추출용액 $1.0 \mathrm{~mL}$ 에 $100 \mathrm{mg} / \mathrm{mL}$ IS $0.1 \mathrm{~mL}$, 포화
탄산나트륨 $0.5 \mathrm{~mL}$, acetonitrile $0.5 \mathrm{~mL}$ 를 잘 혼합 한 뒤 $1 \%$ dansyl chloride $1.0 \mathrm{~mL}$ 용액을 천천히 넣어주고 vortexing하였 다. 잘 혼합된 용액은 $45^{\circ} \mathrm{C}$ 에서 2 시간 동안 유도체화를 거친 후 $10 \%$ proline $0.5 \mathrm{~mL}$ 를 넣어 여분의 dansyl chloride를 제거 하고, 유도체화된 BAs 용액에 diethyl ether $5 \mathrm{~mL}$ 로 분액 한 뒤, 유기용매층을 취하여 감압농축 하였다. Acetonitrile $1.0 \mathrm{~mL}$ 로 재 용해 한 뒤 $0.22 \mu \mathrm{m}$ syringe filter로 여과 후 기기분석에 사용 하였다. 회수율 시험은 시료 추출용액에 표준물질의 농도가 각 각 $10 \mathrm{mg} / \mathrm{kg}$ 가 되도록 spiking하고, 동일한 시험법을 거친 후 측정하였다.

기기분석. 흡광검출기 및 형광검출기가 장착된 UPLC (Acquity UPLC, Waters, Milford, USA)를 사용하였고, 정제칼럼은 $\mathrm{BEH}$ $\mathrm{C}_{18}(1.7 \mu \mathrm{m}, 2.1 \times 50 \mathrm{~mm}$, Waters, Dublin, Ireland)을 사용하였 다. 칼럼온도를 $37^{\circ} \mathrm{C}$ 로 유지하면서 $0.5 \mathrm{~mL} / \mathrm{min}$ 유속으로 $50 \%$ acetonitrile/water (A)과 $100 \%$ acetonitrile (B)을 gradient $(0-5$ $\min , 100 \%$ A; 5-6 min, 95\% A; 6-7 min, 85\% A; 7-8 min, $60 \% \mathrm{~A} ; 8-10 \mathrm{~min}, 30 \% \mathrm{~A} ; 10-11 \mathrm{~min}, 10 \% \mathrm{~A})$ 조건을 설정 하였고, 흡광검출 파장은 $225 \mathrm{~nm}$, 형광검출 조건은 exitation $325 \mathrm{~nm}$, emission $525 \mathrm{~nm}$ 로 설정하여 측정하였다.

\section{결과 및 고찰}

분석회수율 및 정량한계. $\mathrm{AGM}$ 은 형광검출결과를 정량분석에 이용하였으며, 나머지 9종의 $\mathrm{BAs}$ 는 모두 흡광 검출결과를 정 량분석에 사용하였고, 형광분석결과는 정성적인 결과를 보조하 는 수단으로 사용하였다(Fig. 2). $\mathrm{AGM}$ 을 제외한 9종 $\mathrm{BAs}$ 에 대 한 본 시험법의 회수율은 모든 발효식품 시료에서 $65.7-125 \%$ 로 나타났으나, 장아찌시료에서 $\mathrm{AGM}$ 의 회수율은 $<60 \%$ 로 확 인되어, $\mathrm{AGM}$ 에 대한 정량 분석시험은 실시하지 않았다. 또한, $\mathrm{BAs}$ 정량한계 $(\mathrm{LOQ}, 10 \times \mathrm{S} / \mathrm{N})$ 는 $0.5-5.0 \mathrm{mg} / \mathrm{kg}$ 로 확인되었다 (Table 1).

발효식품 중 biogenic amine 잔류. Scombrotoxin으로 불리는 $\mathrm{HIS}$ 외에 TYR, PUT, CAD, PHE등 5종은 독성발현 가능성으로 인해 발효식품 중 주요 잔류분석 조사대상(Beneduce 등, 2010) 이며, 본 분석에서도 이들 5 종을 포함한 총 10 종의 $\mathrm{AGM}$, 

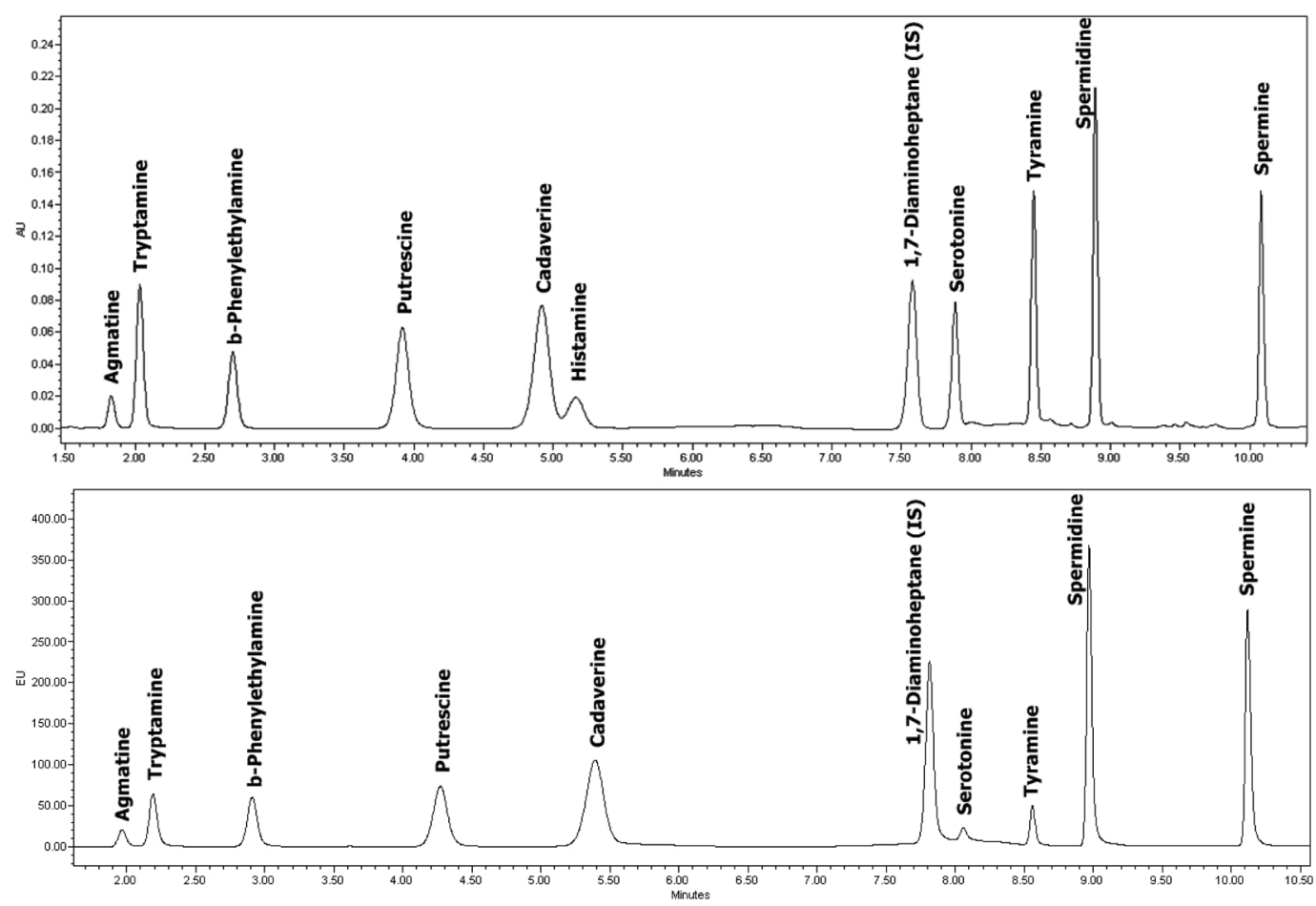

Fig. 2 UPLC-UVD (top) and FLD (bottom) chromatogram of the tested biogenic amines.

TRP, PHE, PUT, CAD, HIS, SER, TYR, SPD, SPM에 대한 잔류분석을 실시하였다(Table 2).

발효진액. 분석대상 발효진액의 원료별 총 $\mathrm{BAs}$ 잔류량은 $<\mathrm{LOQ}$ $-83.7 \mathrm{mg} / \mathrm{kg}$ 으로 높은 잔류량을 나타내지 않았으며, 독성유발 관련 5 종 $\mathrm{BAs}$ 검출비율도 전체 조사시료의 $21 \%$ 이하로 확인 되었다. 발효 진액에서 검출된 독성관련 $\mathrm{BA}$ 는 도라지와 오디 를 이용한 발효진액에서 PUT 한가지만 확인되었으며, 잔류량 또한 각각 $4.7 \mathrm{mg} / \mathrm{kg}$ 과 $1.6 \mathrm{mg} / \mathrm{kg}$ 수준으로 높지 않았다. 특히 ornithine의 decarboxylation으로 생성되는 것으로 알려진 PUT는 발효식품이 아닌 과일, 채소를 이용한 주스, 넥타 등에서도 낮 은 농도로 잔류하는 것으로 알려져 있어(Silla-Santos, 1996;
Mangani 등, 2005), 발효진액에서 검출되는 PUT가 발효작용에 의해 생성되지 않았을 가능성도 있는 것으로 판단된다. $\mathrm{AGM}$ 은 조사대상 진액류의 $60 \%$ 시료에서 검출되었으며, 그 잔류량 은 도라지 $17.7 \mathrm{mg} / \mathrm{kg}$, 오미자 $7.5 \mathrm{mg} / \mathrm{kg}$, 매실 $48.5 \mathrm{mg} / \mathrm{kg}$, 블 루베리 $83.7 \mathrm{mg} / \mathrm{kg}$, 솔잎 $14.9 \mathrm{mg} / \mathrm{kg}$, 오디 $20.1 \mathrm{mg} / \mathrm{kg}$, 복분자 $29.5 \mathrm{mg} / \mathrm{kg}$, 산머루 $6.8 \mathrm{mg} / \mathrm{kg}$ 으로 확인되었다. 이외에 매실과 오디 진액에서 $\mathrm{SPD}$ 가 $2.0 \mathrm{mg} / \mathrm{kg}$ 과 $1.9 \mathrm{mg} / \mathrm{kg}$ 이 확인되었고, 옻 나무와 석류 진액에서는 조사대상 10 종 $\mathrm{BAs}$ 모두 불검출 되었 다(Table 2).

막걸리. 솔잎, 울금, 가시오가피, 인삼을 각각 첨가하여 제조한 막걸리에서 발견된 BAs는 AGM, PUT, TYR 3종 이었으며,

Table 1 Detection limits and recovery rates of ten biogenic amines

\begin{tabular}{|c|c|c|c|c|c|c|}
\hline & \multirow{2}{*}{$\begin{array}{c}\text { LOQ } \\
(\mathrm{mg} / \mathrm{kg})\end{array}$} & \multicolumn{5}{|c|}{ Recovery rates (average $\pm \mathrm{SD}, \%$ )* } \\
\hline & & Picked vegetable & $\begin{array}{c}\text { Fermented vegetable } \\
\text { extracts }\end{array}$ & $\begin{array}{c}\text { Rice wine } \\
\text { (Makgeolli) }\end{array}$ & Black garlic & Fermented tea \\
\hline AGM & 5.0 & $<60.0$ & $73.9 \pm 7.2$ & $84.5 \pm 7.8$ & $110 \pm 7.6$ & $125 \pm 9.4$ \\
\hline TRP & 1.0 & $82.9 \pm 5.3$ & $93.0 \pm 6.4$ & $97.4 \pm 7.7$ & $101 \pm 9.8$ & $97.5 \pm 6.2$ \\
\hline PHE & 1.0 & $102 \pm 8.5$ & $114 \pm 8.3$ & $99.2 \pm 6.3$ & $102 \pm 8.1$ & $105 \pm 4.5$ \\
\hline PUT & 1.0 & $107 \pm 9.2$ & $80.2 \pm 7.7$ & $98.9 \pm 5.2$ & $89.6 \pm 8.3$ & $101 \pm 5.8$ \\
\hline CAD & 0.5 & $111 \pm 9.7$ & $109 \pm 6.7$ & $114 \pm 9.3$ & $102 \pm 9.4$ & $101 \pm 7.6$ \\
\hline HIS & 2.0 & $100 \pm 6.7$ & $72.3 \pm 6.9$ & $120 \pm 10.9$ & $108 \pm 7.3$ & $120 \pm 8.8$ \\
\hline SER & 2.0 & $124 \pm 10.3$ & $76.1 \pm 5.2$ & $112 \pm 10.7$ & $112 \pm 10.5$ & $76.5 \pm 7.9$ \\
\hline TYR & 1.0 & $99.8 \pm 8.6$ & $119 \pm 9.4$ & $99.1 \pm 8.5$ & $98.8 \pm 7.7$ & $96.3 \pm 4.8$ \\
\hline SPD & 1.0 & $94.3 \pm 7.9$ & $68.9 \pm 7.8$ & $97.8 \pm 6.3$ & $94.9 \pm 5.8$ & $79.5 \pm 5.7$ \\
\hline SPM & 1.5 & $82.5 \pm 6.1$ & $65.7 \pm 8.9$ & $85.5 \pm 7.9$ & $81.5 \pm 6.2$ & $71.0 \pm 4.9$ \\
\hline
\end{tabular}

*Recovery rates were measured on $10 \mathrm{mg} / \mathrm{kg}$ with five replications. AGM: agmatine, TRP: tryptamine, PHE: $\beta$-phenylethylamine, PUT: putrescine, CAD: cadaverine, HIS: histamine, SER: serotonine, TYR: tyramine, SPD: spermidine, SPM: spermine 
Table 2 Biogenic amines concentration (average $\pm \mathrm{SE}, \mathrm{mg} / \mathrm{kg}$ ) of minor Korean plant-based fermented foods

\begin{tabular}{|c|c|c|c|c|c|c|c|c|c|c|c|}
\hline & Sources & AGM & TRP & PHE & PUT & CAD & HIS & SER & TYR & SPD & SPM \\
\hline \multirow{10}{*}{$\begin{array}{l}\text { Fermented } \\
\text { vegetable } \\
\text { extract }\end{array}$} & Lacquer tree $(n=3)$ & $<\mathrm{LOQ}$ & $<\mathrm{LOQ}$ & $<\mathrm{LOQ}$ & $<\mathrm{LOQ}$ & $<\mathrm{LOQ}$ & $<\mathrm{LOQ}$ & $<$ LOQ & $<\mathrm{LOQ}$ & $<\mathrm{LOQ}$ & $<\mathrm{LOQ}$ \\
\hline & Bellflower root $(n=3)$ & $17.7 \pm 8.8$ & $<\mathrm{LOQ}$ & $<\mathrm{LOQ}$ & $4.7 \pm 1.7$ & $<\mathrm{LOQ}$ & $<\mathrm{LOQ}$ & $<\mathrm{LOQ}$ & $<\mathrm{LOQ}$ & $<\mathrm{LOQ}$ & $<\mathrm{LOQ}$ \\
\hline & Schisandra $(n=3)$ & $7.5 \pm 5.1$ & $<\mathrm{LOQ}$ & $<\mathrm{LOQ}$ & $<\mathrm{LOQ}$ & $<\mathrm{LOQ}$ & $<\mathrm{LOQ}$ & $<\mathrm{LOQ}$ & $<\mathrm{LOQ}$ & $<\mathrm{LOQ}$ & $<$ LOQ \\
\hline & Japanese apricot $(n=3)$ & $48.5 \pm 10.8$ & $<\mathrm{LOQ}$ & $<\mathrm{LOQ}$ & $<\mathrm{LOQ}$ & $<\mathrm{LOQ}$ & $<\mathrm{LOQ}$ & $<\mathrm{LOQ}$ & $<\mathrm{LOQ}$ & $2.0 \pm 1.5$ & $<\mathrm{LOQ}$ \\
\hline & Blueberry $(\mathrm{n}=3)$ & $83.7 \pm 81.2$ & $<\mathrm{LOQ}$ & $<\mathrm{LOQ}$ & $<\mathrm{LOQ}$ & $<\mathrm{LOQ}$ & $<\mathrm{LOQ}$ & $<\mathrm{LOQ}$ & $<\mathrm{LOQ}$ & $<\mathrm{LOQ}$ & $<\mathrm{LOQ}$ \\
\hline & Pomegranate $(n=3)$ & $<\mathrm{LOQ}$ & $<\mathrm{LOQ}$ & $<\mathrm{LOQ}$ & $<\mathrm{LOQ}$ & $<\mathrm{LOQ}$ & $<\mathrm{LOQ}$ & $<\mathrm{LOQ}$ & $<\mathrm{LOQ}$ & $<\mathrm{LOQ}$ & $<\mathrm{LOQ}$ \\
\hline & Pine needles $(n=4)$ & $14.9 \pm 8.9$ & $<\mathrm{LOQ}$ & $<\mathrm{LOQ}$ & $<\mathrm{LOQ}$ & $<\mathrm{LOQ}$ & $<\mathrm{LOQ}$ & $<$ LOQ & $<\mathrm{LOQ}$ & $<\mathrm{LOQ}$ & $<\mathrm{LOQ}$ \\
\hline & Mulberry $(n=3)$ & $20.1 \pm 17.5$ & $<\mathrm{LOQ}$ & $<\mathrm{LOQ}$ & $1.6 \pm 1.1$ & $<\mathrm{LOQ}$ & $<\mathrm{LOQ}$ & $<\mathrm{LOQ}$ & $<\mathrm{LOQ}$ & $1.9 \pm 1.4$ & $<\mathrm{LOQ}$ \\
\hline & Rubus coreanus $(\mathrm{n}=3)$ & $29.5 \pm 8.4$ & $<\mathrm{LOQ}$ & $<\mathrm{LOQ}$ & $<\mathrm{LOQ}$ & $<\mathrm{LOQ}$ & $<\mathrm{LOQ}$ & $<$ LOQ & $<\mathrm{LOQ}$ & $<\mathrm{LOQ}$ & $<\mathrm{LOQ}$ \\
\hline & Wild grape $(n=2)$ & $6.8 \pm 4.3$ & $<$ LOQ & $<\mathrm{LOQ}$ & $<\mathrm{LOQ}$ & $<\mathrm{LOQ}$ & $<$ LOQ & $<$ LOQ & $<\mathrm{LOQ}$ & $<\mathrm{LOQ}$ & $<\mathrm{LOQ}$ \\
\hline \multirow{5}{*}{$\begin{array}{l}\text { Rice wine } \\
\text { (Makgeolli) }\end{array}$} & Pine needles $(n=3)$ & $668 \pm 189$ & $<\mathrm{LOQ}$ & $<\mathrm{LOQ}$ & $7.1 \pm 2.8$ & $<\mathrm{LOQ}$ & $<\mathrm{LOQ}$ & $<\mathrm{LOQ}$ & $<\mathrm{LOQ}$ & $<\mathrm{LOQ}$ & $<\mathrm{LOQ}$ \\
\hline & Tumeric $(n=3)$ & $470 \pm 128$ & $<\mathrm{LOQ}$ & $<\mathrm{LOQ}$ & $6.4 \pm 3.2$ & $<\mathrm{LOQ}$ & $<\mathrm{LOQ}$ & $<$ LOQ & $10.6 \pm 7.6$ & $<\mathrm{LOQ}$ & $<\mathrm{LOQ}$ \\
\hline & Gasi-Ogalipy $(\mathrm{n}=3$ ) & $782 \pm 150$ & $<\mathrm{LOQ}$ & $<\mathrm{LOQ}$ & $29.0 \pm 12.8$ & $<\mathrm{LOQ}$ & $<\mathrm{LOQ}$ & $<\mathrm{LOQ}$ & $40.1 \pm 8.8$ & $<\mathrm{LOQ}$ & $<\mathrm{LOQ}$ \\
\hline & Ginseng $(n=3)$ & $499 \pm 121$ & $<\mathrm{LOQ}$ & $<\mathrm{LOQ}$ & $7.9 \pm 5.0$ & $<\mathrm{LOQ}$ & $<\mathrm{LOQ}$ & $<\mathrm{LOQ}$ & $<\mathrm{LOQ}$ & $<\mathrm{LOQ}$ & $<\mathrm{LOQ}$ \\
\hline & Non-addition $(\mathrm{n}=2)$ & $530 \pm 184$ & $<\mathrm{LOQ}$ & $<\mathrm{LOQ}$ & $3.2 \pm 1.1$ & $<\mathrm{LOQ}$ & $<\mathrm{LOQ}$ & $<\mathrm{LOQ}$ & $<\mathrm{LOQ}$ & $<\mathrm{LOQ}$ & $<\mathrm{LOQ}$ \\
\hline \multirow{10}{*}{$\begin{array}{c}\text { Pickled } \\
\text { vegetable }\end{array}$} & Perilla leaf $(1$ & N.T. ${ }^{*}$ & $21.3 \pm 8.3$ & $8.8 \pm 6.2$ & $90.4 \pm 30$ & 1 & $25.0 \pm 9.8$ & $<\mathrm{LOQ}$ & $28.0 \pm 8.5$ & $4.0 \pm 2.8$ & $3.2 \pm 1.5$ \\
\hline & Mulberry leaf $(n=3)$ & N.T. & $<\mathrm{LOQ} 2$ & $21.9 \pm 12.3$ & $70.7 \pm 28.5$ & $3.6 \pm 1.8$ & $201 \pm 81.3$ & $<\mathrm{LOQ}$ & $98.4 \pm 25.8$ & $<\mathrm{LOQ}$ & $11.7 \pm 2.5$ \\
\hline & Soy leaf $(n=3)$ & N.T. & $58.0 \pm 25.9$ & $11.8 \pm 6.8$ & $356 \pm 145$ & $17.5 \pm 8.1$ & $376 \pm 102$ & $<\mathrm{LOQ}$ & $453 \pm 135$ & $<\mathrm{LOQ}$ & $2.2 \pm 1.1$ \\
\hline & Pepper $(n=4)$ & N.T. & $16.2 \pm 8.9$ & $2.7 \pm 1.8$ & $68.4 \pm 20.1$ & $12.0 \pm 3.5$ & $53.9 \pm 12.5$ & $<\mathrm{LOQ}$ & $37.4 \pm 20.1$ & $16.5 \pm 4.2$ & $16.2 \pm 3.5$ \\
\hline & Garlic $(n=4)$ & N.T. & $47.4 \pm 18.7$ & $<\mathrm{LOQ}$ & $17.0 \pm 8.5$ & $11.6 \pm 3.8$ & $24.9 \pm 8.7$ & $<\mathrm{LOQ}$ & $10.4 \pm 6.2$ & $<\mathrm{LOQ}$ & $5.3 \pm 2.4$ \\
\hline & Persimmon $(\mathrm{n}=2)$ & N.T. & $<\mathrm{LOQ}$ & $<\mathrm{LOQ}$ & $10.3 \pm 6.3$ & $5.8 \pm 2.6$ & $8.8 \pm 4.2$ & $<$ LOQ & $6.9 \pm 2.5$ & $3.7 \pm 2.1$ & $10.8 \pm 3.6$ \\
\hline & Onion $(n=3)$ & N.T. & $<\mathrm{LOQ}$ & $<\mathrm{LOQ}$ & $9.5 \pm 3.2$ & $13.6 \pm 4.2$ & $10.1 \pm 4.2$ & $<\mathrm{LOQ}$ & $6.6 \pm 3.7$ & $5.2 \pm 2.8$ & $14.7 \pm 4.2$ \\
\hline & Deodeok $(n=2)$ & N.T. & $<\mathrm{LOQ}$ & $<\mathrm{LOQ}$ & $29.3 \pm 7.8$ & $12.6 \pm 2.8$ & $15.4 \pm 5.0$ & $<\mathrm{LOQ}$ & $30.4 \pm 12.7$ & $5.1 \pm 2.7$ & $7.2 \pm 1.8$ \\
\hline & Oriental melon $(\mathrm{n}=2)$ & N.T. & $26.5 \pm 18.2$ & $<\mathrm{LOQ}$ & $<$ LOQ & $7.6 \pm 3.3$ & $6.0 \pm 2.2$ & $<\mathrm{LOQ}$ & $5.6 \pm 3.2$ & $3.2 \pm 1.8$ & $3.1 \pm 1.6$ \\
\hline & Plum $(n=2)$ & N.T. & $<\mathrm{LOQ}$ & $<\mathrm{LOQ}$ & $17.3 \pm 6.4$ & $13.5 \pm 3.8$ & $9.3 \pm 4.1$ & $<$ LOQ & $6.7 \pm 2.9$ & $3.2 \pm 1.8$ & $1.8 \pm 0.9$ \\
\hline Black garlic & Garlic $(\mathrm{n}=10)$ & $<\mathrm{LOQ}$ & $<\mathrm{LOQ}$ & $<\mathrm{LOQ}$ & $<\mathrm{LOQ}$ & $<\mathrm{LOQ}$ & $<\mathrm{LOQ}$ & $<\mathrm{LOQ}$ & $<\mathrm{LOQ}$ & $<\mathrm{LOQ}$ & $<\mathrm{LOQ}$ \\
\hline Fermented tea & Tea leaf $(n=15)$ & $15.8 \pm 12.1$ & $<\mathrm{LOQ}$ & $<\mathrm{LOQ}$ & $12.4 \pm 4.4$ & $<\mathrm{LOQ}$ & $<\mathrm{LOQ}$ & $<\mathrm{LOQ}$ & $13.0 \pm 5.4$ & $22.9 \pm 8.1$ & $31.3 \pm 19.6$ \\
\hline
\end{tabular}

*N.T. means not tested. AGM: agmatine, TRP: tryptamine, PHE: $\beta$-phenylethylamine, PUT: putrescine, CAD: cadaverine, HIS: histamine, SER: serotonine, TYR: tyramine, SPD: spermidine, SPM: spermine

$\mathrm{AGM}$ 과 PUT는 발효첨가물의 여부와 무관하게 모든 분석시료 에서 검출되었고, $\mathrm{AGM}$ 은 $100 \mathrm{mg} / \mathrm{kg}$ 이상 고농도가 검출 (470-782 mg/kg) 되었다. PUT는 가시오가피를 첨가한 막걸리에 서 $29.0 \mathrm{mg} / \mathrm{kg}$ 검출되었으며, 솔잎, 울금, 인삼을 첨가하여 제조 된 막걸리는 식물추출물을 첨가하지 않은 막걸리와 유사한 수 준의 잔류량 $(3.2-7.9 \mathrm{mg} / \mathrm{kg})$ 을 나타내었다. 또한, TYR은 가시오 가피와 울금이 각각 첨가된 막걸리시료에서 $10.6-40.1 \mathrm{mg} / \mathrm{kg}$ 수 준으로 검출되었다. 솔잎, 울금, 가시오가피, 인삼과 같은 발효 첨가물에 의해 $\mathrm{AGM}$ 의 막걸리 중 잔류량이 증가하거나 감소하 는 영향을 받는 것으로 판단하기에는 자료가 부족하며, 특히 PUT와 TYR은 Kim 등(2011)이 보고한 바와 같이 제조 후 보 관조건에 따라 생성 가능하므로 식물 추출첨가물에 의한 영향 여부에 대해서는 보완연구가 필요하다(Table 2).

장아찌류. 장아찌는 제조공정상 발효식품으로 분류하지 않지만, 본 연구에 사용된 시료는 된장, 간장 등을 사용하여 제조되기 때문에, 장류의 $\mathrm{BAs}$ 가 제조공정 중 장아찌에 이행될 수 있을 가능성이 높아, 장아찌류에 대한 $\mathrm{BAs}$ 잔류 분석을 수행하게 되 었다. 본 시험에서 $\mathrm{AGM}$ 은 회수율 시험성적이 $60 \%$ 미만으로 확인되어, 정량분석대상에서 제외하였다. 총 $\mathrm{BAs}$ 함량이 가장 높은 것으로 조사된 콩잎장아찌는 총 $\mathrm{BAs}$ 함량이 $1,274 \mathrm{mg} / \mathrm{kg}$ 이었으며, TYR $453 \mathrm{mg} / \mathrm{kg}$, HIS $376 \mathrm{mg} / \mathrm{kg}$, PUT $356 \mathrm{mg} / \mathrm{kg}$,
TRP $58.0 \mathrm{mg} / \mathrm{kg}, \quad$ CAD $17.5 \mathrm{mg} / \mathrm{kg}, \quad$ PHE $11.8 \mathrm{mg} / \mathrm{kg}, \quad$ SPM $2.2 \mathrm{mg} / \mathrm{kg}$ 순으로 조사되었고, 독성유발 가능성이 있는 $\mathrm{PHE}$, $\mathrm{PUT}, \mathrm{CAD}, \mathrm{HIS}, \mathrm{TYR} 5$ 종 $\mathrm{BAs}$ 의 잔류분포가 $95 \%$ 로 확인되 었다. 또한, 뽕잎의 경우에도 총 BAs 함량이 $407 \mathrm{mg} / \mathrm{kg}$ 으로 높 았으며, HIS $201 \mathrm{mg} / \mathrm{kg}$, TYR $98.4 \mathrm{mg} / \mathrm{kg}$, PUT $70.7 \mathrm{mg} / \mathrm{kg}$, PHE $21.9 \mathrm{mg} / \mathrm{kg}, \mathrm{SPM} 11.7 \mathrm{mg} / \mathrm{kg}, \mathrm{CAD} 3.6 \mathrm{mg} / \mathrm{kg}$ 의 순으로 조사되었고, 독성 유발가능 5 종 $\mathrm{BAs}$ 잔류분포는 $97 \%$ 로 확인 되었다. 고추와 깻잎장아찌의 총 $\mathrm{BAs}$ 함량은 $223 \mathrm{mg} / \mathrm{kg}$ 와 $213 \mathrm{mg} / \mathrm{kg}$ 으로 각각 조사되었고, 독성 유발가능 5 종 $\mathrm{BAs}$ 비율 은 각각 $78 \%$ 와 $86 \%$ 로 확인되었으며, PUT 잔류량이 $90.4 \mathrm{mg}$ $\mathrm{kg}$ 과 $68.4 \mathrm{mg} / \mathrm{kg}$ 으로 가장 높은 것으로 조사되었다. 그리고, 이 들의 총 $\mathrm{BAs}$ 함량이 $100 \mathrm{mg} / \mathrm{kg}$ 을 초과하는 시료는 마늘과 더 덕장아찌로 조사되었고, 이들의 독성 유발가능 5 종 $\mathrm{BAs}$ 잔류 분포는 각각 $54 \%$ 와 $87 \%$ 로 확인되었다. 총 $\mathrm{BAs}$ 함량이 $100 \mathrm{mg} / \mathrm{kg}$ 미만인 시료에서는 양파, 참외, 자두, 감의 순서로 총 $\mathrm{BAs}$ 함량이 높게 나타났고, 각각 $59.7,52.0,51.8,46.3 \mathrm{mg} / \mathrm{kg}$ 으로 조사되었다. 이들의 독성 유발가능 5 종 $\mathrm{BAs}$ 잔류비율은 각각 $66,36,90,68 \%$ 로 확인되었고, SER은 모든 시료에서 검 출되지 않았으며, PHE는 깻잎, 뽕잎, 콩잎, 고추장아찌에서만 검출되었다. 하지만, 총 $\mathrm{BAs}$ 잔류량과 독성유발가능 5 종 $\mathrm{BAs}$ 잔류량간의 정량적 상관성은 관찰되지 않았다 (Table 2). 
흑마늘. 흑마늘은 마늘을 $60^{\circ} \mathrm{C}$ 이상의 고온에서 열숙성시켜 갈 변반응으로 얻어지는 1 단계와 $40-50^{\circ} \mathrm{C}$ 및 실온에서 약 7 일간 안정화 시키는 2 단계를 거쳐 일반적으로 제조되고 있다. 이로 인해 흑마늘 제품을 열숙성 식품으로 분류하기도 하지만, 최근 일부 연구자들은 발효가 진행 된 발효식품으로도 설명하고 있 다(You 등 2011, Kim 등 2012). 따라서, 본 연구에서는 학계 에서 발효여부에 대한 논란은 있지만, 흑마늘 제조공정이 아직 표준화 되어 있지 않고, 생산자 및 소비자들이 흑마늘을 발효 식품으로 인지하고 있음을 감안하여 흑마늘에서의 BAs 잔류분 석을 실시하고, 그 결과를 제시하고자 하였다. 분석대상 흑마늘 은 제조사별 통흑마늘 5 종 및 액상흑마늘 5 종에 대한 잔류분석 을 실시하였으며, 분석대상에서 $\mathrm{BAs}$ 는 모두 검출되지 않았다 (Table 2).

발효차 분석대상 시료에서 $\mathrm{SPM}$ 이 $93 \%, \mathrm{SPD}$ 가 $73 \%$ 순으로 높게 검출되었으며, 평균 잔류농도는 $31.3 \mathrm{mg} / \mathrm{kg}$ 과 $22.9 \mathrm{mg} / \mathrm{kg}$ 으로 확인되었다. 이외에 TYR, PUT, AGM 의 순으로 검출빈 도가 높게 나타났으며 $20.0,13.3,6.7 \%$ 로 각각 조사되었다. 본 시험에서의 대조구로 비발효 녹차 2종 및 현미녹차, 둥글레차 에 대한 $\mathrm{BAs}$ 잔류분석을 실시하였으며, 그 결과 비발효 녹차 에서는 SPM만 $23.6 \mathrm{mg} / \mathrm{kg}$ 수준으로 확인되었고, 현미녹차에서 는 $\mathrm{SPD} 12.1 \mathrm{mg} / \mathrm{kg}$ 와 $\mathrm{SPM} 17.3 \mathrm{mg} / \mathrm{kg}$ 2종이 검출되었다. 반 면, 둥글레차에서는 BAs가 검출되지 않았다 (Table 2).

위의 결과를 종합하면, 일부 발효 농식품 시료에서 상대적으 로 높은 함량의 $\mathrm{BAs}$ 가 검출되기도 하였으나, 이들 식품의 $\mathrm{BAs}$ 잔류위해성 규정을 위해서는 전국 단위의 $\mathrm{BAs}$ 잔류 모니터링 및 이들 결과에 근거한 위해성 평가연구가 필요할 것이다.

\section{초 록}

본 연구에서는 식물을 원료로 하여 제조된 발효식품들 중 지금 까지 $\mathrm{BAs}$ 에 대한 잔류분석연구가 진행되지 못하였던, 식품군들 에 대해 BAs 잔류분석을 실시하였다. 조사대상 식품군으로는 식물 발효진액, 장아찌, 막걸리, 흑마늘, 발효차를 선정하였으며, 발효진액, 막걸리, 흑마늘, 발효차는 HIS, TYR, PUT, CAD, PHE 5종 BAs 의 총 함량이 $1,000 \mathrm{mg} / \mathrm{kg}$ 을 초과하지 않았으나, 장아찌시료에서는 $19.2-1,214 \mathrm{mg} / \mathrm{kg}$ 으로 검출되었다. 이러한, 장 아찌류의 고잔류 $\mathrm{BAs}$ 는 간장과 된장 등 원료 장류에 의한 영 향이 높을 것으로 사료되며, 장아찌에 대한 $\mathrm{BAs}$ 연구는 원료 및 제조 특성별 잔류 분석연구가 보완되어야 할 것이다.

Keywords 바이오제닉아민 · 발효식품 · 잔류
감사의 글 본 연구는 농촌진흥청 공동연구사업 (PJ007425)의 지원으로 수 행되었습니다.

\section{References}

Bardocz A (1995) Polyamines in food and their consequences for food quality and human health. Trends Food Sci Technol 6, 341-6.

Beneduce L, Romano A, Capozzi V, Lucas P, Barnavon L, Bach B et al. (2010) Biogenic amine in wines. Ann Microbiol 60, 573-8.

Cho T-Y, Han G-H, Bahn K-N, Son Y-W, Jang M-R, Lee C-H et al. (2006) Evalution of biogenic amines in Korean commercial fermented foods. Kor J Food Sci Technol 39, 541-5.

Chong CY, Abu Baker F, Russly AR, Jamilah B, and Mahyudin NA (2011) The effects of food processing on biogenic amines formation. Int Food Res $J$ 18, 867-76.

Choi JY, Hong SW, and Chung KS (2012) Selection of biogenic aminereducing microorganisms from a traditional Korean-style fermented food, Cheonggukjang. Kor J Food Sci Technol 44, 196-201.

Greif G, Greifova M, and Drdak M (1997) Determination of biogenic amines in foods of animal origin by HPLC. Potrav Vedy 15, 119-29.

Halasz A, Barath A, Simon-Sarkadi L, and Holzapfel W (1994) Biogenic amines and their production by microrganisms in food. Trends Food Sci Technol 5, 42-9.

Hu Y, Huang Z, Li J, and Yang H (2012) Concentration of biogenic amines in fish, squid and octopus and their changes during storage. Food Chem 135, 2604-11.

Karovicova J and Kohajdova Z (2005) Biogenic amines in Food. Chem Pap 59, 70-9.

Kim J-H, Ahn H-J, Kim D-H, Jo C, Cha B-S, and Byun M-W (2002) Effect of gamma irradiation on biogenic amines levels in Doenjang during fermation. J Kor Soc Food Sci Nutr 31, 713-6.

Kim JY, Kim D, Park P, Kang H-I, Ryu EK, and Kim SM (2011) Effects of storage temperature and time on the biogenic amine content and microflora in Korean turbid rice wine, Makgeolli. Food Chem 128, 87 92.

Kim M-S, Kim M-J, Bang W-S, Kim K-S, and Park S-S (2012) Determination of S-Allyl-L-cystein, diallyl disulfide and total amino acids of black garlic after spontaneous short-term fermentation. J Korean Soc Food Sci Nutr 41, 661-5.

Lehane L and Olley J (2000) Histamine fish poisoning revisited. Int J Food Microbiol 58, 1-37.

Mangani S, Guerrini S, Granchi L, and Vincenzini M (2005) Putrescine accumulation in wine: Role of Oenococcus oeni. Curr Microbiol 51, 610 .

Silla-Santos MH (1996) Biogenic amines: their importance in foods. Int $J$ Food Microbiol 29, 213-31.

You BR, Kim HR, Kim MJ, and Kim MR (2011) Comparison of the quality characteristics and antioxidant activities of the commercial black garlic and lab-prepared fermented and aged black garlic. J Korean Soc Food Sci Nutr 40, 366-71. 